\title{
Liderazgo e Innovación para un Mundo más Sostenible
}

\author{
Goretti Cabaleiro ${ }^{1}$, Alejandro Jiménez ${ }^{1}$, John Miles ${ }^{2}$, Roberto Horta ${ }^{2 *}$
}

\section{Presentación}

El siguiente número se compone de 14 artículos presentados en el marco del 21 Foro Mundial de la Asociación Internacional de Escuelas de Negocios Jesuitas (IAJBS) y la 18 Conferencia Anual de los Académicos de Escuelas de Negocios Jesuitas (CJBE). Ambos eventos se realizaron simultáneamente en la Universidad Católica del Uruguay en julio de 2015 y participaron 254 personas provenientes de 49 universidades y 22 países. El lema general del encuentro fue "Liderazgo e Innovación para la Sostenibilidad Global" y las conferencias, paneles y presentaciones se distribuyeron principalmente en 4 ejes temáticos:

a) El estilo de liderazgo jesuita como aporte al desarrollo sostenible.

Se reconoce que el liderazgo en las organizaciones es un aspecto clave en el desarrollo de las economías y sociedades del siglo XXI. Los directivos y demás personas que intervienen en las organizaciones tienen que ser capaces de evaluar los aspectos sociales, políticos, económicos, legales, interculturales o tecnológicos relacionados con su organización y la misión que tienen en la sociedad. En general, cuando se habla de "liderazgo al estilo jesuita" (Lowney, 2004) se piensa en un líder transformador de organizaciones que con su actuar, contribuye a lograr una sociedad más justa y humana. Su liderazgo se basa en cuatro pilares - que desarrolla ampliamente Lowney en su bibliografía- el conocimiento de sí mismo, el ingenio, el amor y el heroísmo. Se trata de un cambio de paradigma que toma en cuenta las diferentes inteligencias "múltiples" de Howard Gardner (1983), la "emocional" de Daniel Goleman (1995) y coloca como centro al individuo que, primero debe autoliderarse a sí mismo, para luego poder liderar a otros. Es en ese "liderazgo de otros" (el tradicional) que se pueden -en un mundo cada vez más globalizado e interrelacionado- hacer más competitivas y sostenibles las organizaciones.

b) Innovación en la pedagogía de la enseñanza de los negocios en universidades de inspiración jesuita

Se afirma en la necesidad de asistir a un proceso de innovación en las escuelas de negocios que implique una evolución pedagógica y profesional con el fin de dar respuesta a las demandas que surgen de la evolución de la sociedad (AACSB, 2011). Las innovaciones se caracterizan por una diversidad de formas, modalidades y alcances que implican cambios en las actividades y en las actitudes. En consecuencia, se requiere tecnología, metodologías novedosas de enseñanza-aprendizaje, nuevas capacidades para los alumnos y actualización de las competencias pedagógicas y disciplinares de los académicos (Fox y Vikers, 2010; Fox y Cox, 2004, Roberson y Franchini, 2014; Michaelsen y Sweet, 2008). Se abordan cuestiones como los nuevos paradigmas en la enseñanza de negocios y metodologías de enseñanza-aprendizaje en el contexto actual. Además, se reconocen los elementos de soporte necesarios para obtener un aprendizaje de calidad. Se aborda la educación virtual, reflexionando sobre los impactos de esta modalidad en el proceso de enseñanza-aprendizaje. La pedagogía, como arte y ciencia de enseñar, no puede reducirse simplemente a una metodología; debe incluir una perspectiva del mundo y una visión de la persona humana que se pretende formar (Kolvenbach, 2001; Nicolas, 2010). En este sentido, es esencial reconocer e identificar los cambios necesarios en los planes y programas de las escuelas de negocios para formar personas capaces de transformar la realidad.

c) Innovación en la relación empresa-universidad de inspiración jesuita.

En un mundo que cambia exponencialmente, el fortalecimiento entre las universidades y las empresas es imprescindible para que la innovación resulte en un desarrollo sostenible. En ese sentido, se busca abordar el desafío de potenciar la relación empresa-universidad en un contexto de cambio acelerado. La generación de conocimiento y la innovación son el resultado de una red compleja de relaciones donde las universidades son un actor más entre otros. En esta perspectiva, la misión de creación y difusión del conocimiento adquiere un nuevo sentido, principalmente en términos estratégicos de cómo desempeñar este rol. Las universidades vienen asumiendo una actitud emprendedora con el objetivo de establecer nuevas formas de relación con todas las partes involucradas en la creación y difusión del conocimiento. En las economías emergentes, el rol emprendedor de las universidades es especialmente relevante, debido a la necesidad de generar una actitud de colaboración entre universidades, gobiernos y empresas. El objetivo es aprovechar el avance exponencial de las tecnologías y transformar las bases económicas de las ciudades, regiones y países. Por lo tanto, las universidades no pueden quedarse simplemente aportando conocimiento técnico, sino que también deben ayudar a reflexionar sobre el sentido y las bases éticas de la innovación con el objeto de favorecer el desarrollo económico.

d) Reflexiones para un mundo más sostenible: experiencias exitosas.

Se busca presentar experiencias concretas de empresas cuyas acciones estén aportando al desarrollo sostenible de Latinoamérica. Los países de la región atraviesan un momento histórico, en que se

(1) Facultad de Economía y Negocios, Universidad Alberto Hurtado, Chile.

(2) Facultad de Ciencias Empresariales, Universidad Católica del Uruguay.

* Autor de correspondencia: rhorta@ucu.edu.uy 
verifican progresos en el área económica, con impactos en diversos temas sociales y una mayor estabilidad política que se ha ido consolidando en los últimos años. A pesar que el crecimiento económico se ha desacelerado en el último tiempo, han ocurrido importantes avances en la reducción de la pobreza extrema, la desnutrición, la mortalidad infantil y la falta de acceso al agua, entre otros aspectos sociales. Es decir, los países de la región han mejorado su sostenibilidad económica y social, pero existen importantes desafíos para el futuro. En general, la región arrastra una oferta educativa de baja calidad y un desempleo de la población. Se apuesta a la incorporación de las mujeres al mercado laboral. Sin embargo, persiste la discriminación basada en el género. Se ha generalizado una política social asistencial y falta implementar programas de protección que busquen eliminar las vulnerabilidades existentes de una manera integral. En dicho marco, existen numerosos casos de empresas y organizaciones que vienen incorporando estrategias y acciones para apoyar un desarrollo sostenible en sus comunidades y regiones. El conocimiento de dichas experiencias, la forma en que se han venido ejecutando, los motivos que las impulsan, etc. son aportes significativos para las escuelas de negocios y otros actores que deseen contribuir en la construcción de una economía sostenible.

\section{La edición especial: Liderazgo e Innovación para un Mundo más Sostenible.}

En el Foro Mundial IAJBS/CJBE 2015 se presentaron 103 artículos de los cuales, 72 fueron aceptados y presentados en las diferentes sesiones de trabajo. Adicionalmente, un equipo de editores del Journal of Technology Management \& Innovation (JOTMI) preseleccionó 29 artículos y finalmente decidió publicar -luego de un proceso de revisión- 14 de ellos agrupados en las siguientes 4 categorías:

a) Formas innovadoras de educación para la sostenibilidad, en la que se abordan experiencias y metodologías innovadoras en la educación de negocios:

El primer trabajo de McGrath (2016) busca derribar el falso paradigma de que gran parte de la relación académica entre universidades del oeste con sus pares de Asia se ha desarrollado desde un modelo de "exportación", asumiendo que los asiáticos son los que tienen que aprender de la educación universitaria de occidente (equivocadamente presumida como superior). Profundiza en los distintos estilos de pensamiento, aprendizaje y comportamiento entre los estudiantes chinos y americanos.

El modelo Ledesma-Kolvenbach (LK), aplicado en algunas escuelas de negocios jesuitas, está alineado con las propuestas de muchas organizaciones internacionales - WEF, UN, OCDE, entre otras - que argumentan que la sostenibilidad, la dignidad humana y el bien común deben ser considerados como elementos claves en la estrategia de la compañía. En esta línea, Aguado y col. (2016) utiliza el método LK para formar personas que puedan dirigir organizaciones con un enfoque de sostenibilidad y no preocupados únicamente por maximizar los beneficios para los accionistas.
El tercer trabajo de López de Viguria y Santomá (2016) centra su análisis en la misión de las escuelas de negocios de UNIJES (Universidades Jesuitas en España). En particular, su estudio se centra en los rasgos que caracterizan el perfil de los egresados de dichas escuelas a juicio de sus directivos (rectores y directores). Para ello, los autores utilizan la metodología del Concept Mapping. Sus resultados revelan algunos rasgos del perfil de los egresados y la manera en que se agrupan en cuatro clústeres: (1) competencias profesionales, (2) competencias intrapersonales, (3) competencias interpersonales y (4) responsabilidad social /sentido de trascendencia. Los resultados de esta investigación pueden considerarse como un aporte original para las escuelas de negocios jesuitas, dado su carácter de instituciones mission-driven.

Los trabajos de Díaz Durán (2016) y Marcillo-Gómez y Desilus (2016) se enfocan en las TICs aplicadas a la educación. En el trabajo de Díaz Durán, se hace una profunda reflexión sobre las tecnologías de la información y las comunicaciones aplicadas a la educación en el marco de la pedagogía ignaciana. En el trabajo de Marcillo-Gómez y Desilus se analiza una experiencia de estudio colaborativo entre estudiantes de diferentes realidades culturales, EEUU y México. Se presenta una aproximación pedagógica al aprendizaje colaborativo internacional utilizando las TICs; especial atención reciben los aspectos culturales a la hora de implementar cursos colaborativos entre estudiantes de distintas nacionalidades. Se concluye que mucho de lo que percibimos, creemos y aprendemos depende del contexto cultural.

b) Casos de liderazgo y empresas sostenibles, donde se presentan experiencias de estilos de liderazgo que apoyan el desarrollo de empresas sostenibles:

En el primer trabajo, Barrientos y Reilly (2016) describen el start-up de "Give Back Box" (GBB). Se trata de una empresa social con sede en Chicago que apoya el reciclaje y la donación a través de un modelo de negocios innovador. GBB provee un mecanismo gratuito a través del cual una persona puede reciclar la caja en la que son embalados los productos que compra. El modelo permite realizar una donación de ropa a instituciones de beneficencia. Con lo anterior, se busca generar un cambio donde el reciclaje y la donación se transforman en parte del proceso de compra.

En el segundo trabajo, González y col. (2016) analizan la sustentabilidad organizacional asociado al alto desempeño. Basan su estudio en 55 empresas uruguayas que han perdurado más de 30 años soportando crisis económicas radicales, importantes cambios políticos, sociales y tecnológicos, pero manteniendo posiciones de primera línea en sus áreas de acción. Se parte de la premisa de que las empresas son organizaciones cuya actividad principal son los negocios. Sin embargo, muchas veces se confunde la relación entre organización y negocios (que suele ser creciente, dinámica y variada). La experiencia muestra que las empresas que permanecen en el tiempo se caracterizan por su capacidad de evolucionar en la relación organización-negocio en tres modalidades principales: cambiando su modelo, integrando nuevos negocios y, en forma más radical, migrando a otros negocios. Los resultados muestran que, además de las competencias de gestión, 
aparecen los valores y las actitudes de la dirección. Por ende, se confirma la importancia que tiene para las escuelas de negocios la formación de líderes conscientes de sus decisiones y la dimensión ética en la experiencia educativa de futuros directivos.

c) Relación entre actores del sistema de innovación, que presenta algunas reflexiones y experiencias que aportan a la construcción de una red de escuelas de negocios y su rol en la promoción de la justicia social.

Brabcatelli y Swirski Souza (2016) analizan la necesaria colaboración entre las empresas, el gobierno y la academia para generar formas innovadoras y éticas de encarar los desafíos de la economía global en forma sostenible. Se discute el rol "profético" que deben cumplir las universidades jesuitas ayudando a identificar e incorporar los aspectos éticos y morales que implica el desarrollo tecnológico, las regulaciones gubernamentales y los negocios. Se presenta una experiencia concreta "doing Brazil in Brazil" en donde las universidades pueden poner en práctica el "triángulo de la innovación". Se trata de un encuentro entre empresarios, académicos y gobierno que trabajan en la generación de soluciones a problemas reales incorporando desde el principio los aspectos éticos y morales.

Dado que las universidades aportan al desarrollo territorial, generando redes de conocimiento e innovación y ayudando a vincular las estrategias a las necesidades territoriales, el objetivo del artículo de Aranguren y col. (2016) es reflexionar sobre el papel que desempeñan las instituciones de investigación académica como agentes de cambio. Los institutos de investigación tienen como objetivo generar nuevos conocimientos, además de ayudar al desarrollo de los territorios. Se presentan dos casos de estudio de investigadores que orientan su investigación activamente hacia acciones transformadoras en dos universidades jesuitas; una en el País Vasco, España y la otra en Uruguay.

d) Innovación en las empresas sociales (economía social) en donde se muestra que el emprendimiento social y las empresas sociales han emergido como una estrategia exitosa que busca el desarrollo sustentable y la justicia social.

Douglas y col. (2016) presentan la experiencia del Global Social Benefit Institute $\left(G S B I^{\circledR}\right)$ de la Universidad de Santa Clara, que busca desarrollar la capacidad del emprendimiento social apoyando a los emprendedores sociales. El GSBI ha trabajado con más de 340 emprendimientos sociales operando en más de 60 países. En particular, se profundiza en la vocación de los emprendedores sociales y en las etapas en las cuales se les puede apoyar.

Los modelos asociativos basados en los principios de la Economía Social, han mostrado que favorecen al reparto equitativo del ingreso y además, promueven la autogestión, la democracia interna, la solidaridad y el desarrollo local. Irigoyen (2016) plantea que la innovación social, empresarial y tecnológica alineadas a los principios de la Economía Social, propician la formación y subsistencia de empresas y colectivos que resuelven sus necesidades individuales y de grupo. En esta línea, Irigoyen presenta el Proyecto de Economía Social Idit Ibero que tiene como objetivo incubar, impulsar y/o consolidar empresas de economía social - preferentemente ubicadas en zonas pobres o vulnerables - incorporando un "componente significativo" de desarrollo tecnológico e innovación.

e) Desarrollo sostenible, en el que se argumenta que el éxito del desarrollo sostenible requiere cambiar el paradigma y las maneras de actuar. Los trabajos en esta sección abordan el tema a nivel micro (empresas), nacional (países) y regional.

Bartesaghi y Pereira (2016) analizan como las iniciativas de integración en América Latina - que han aumentado en los últimos años- están encarando los desafíos que presenta el nuevo orden internacional para el desarrollo sostenible de la región. El enfoque local lo aportan Duran y col. (2016) que analiza el caso de la inversión privada en Uruguay. En los últimos 10 años se ha registrado un crecimiento excepcional de la inversión y que en parte se explica por las políticas de promoción. La pregunta planteada es si la inversión promovida en Uruguay - entre 2010 y 2014 representativa de un elevado porcentaje de la inversión privada ejecutada- estaría impactando en el patrón de innovación y en el mercado de trabajo.

En la actual economía globalizada, la innovación es considerada un elemento clave para la competitividad de las firmas. Por lo tanto, tratar de comprender la manera que innovan las empresas en una realidad concreta, puede ayudar en el diseño de políticas de incentivos adecuadas y eficientes. Abordando el tema desde la perspectiva micro, Silveira y col. (2016) estudian las relaciones que las empresas establecen con los diferentes agentes del Sistema Nacional de Innovación (SNI). Se destaca la importancia de las relaciones que establecen las universidades y los centros tecnológicos con la industria para el fomento de la innovación.

\section{Referencias}

AACSB International. Globalization of Management Education Task Force. (2011). Globalization of Management Education: Changing International Structures, Adaptive Strategies, and the Impact on Institutions: Report of the AACSB International Globalization of Management Education Task Force. Aacsb International (Ed.). Emerald Group Publishing.

Vickers, D., \& Fox, S. (2010). Towards practice-based studies of HRM: an actor-network and communities of practice informed approach. The International Journal of Human Resource Management, 21(6), 899-914.

Fox, S y S. Cox (2004) “Management education and leadership." In Cooper, C.I. (ed.) Leadership and Management in the 21st Century.

Kennedy, D. (2006). Writing and using learning outcomes: a practical guide. University College Cork.

Kolvenbach, P.H. (2001) Alocución en la Universidad Católica de Córdoba, Argentina, con ocasión de la inauguración de la sede rectoral, 12 de noviembre de 2001. 
Lowney, C. (2007). El liderazgo al estilo de los jesuitas. Grupo Editorial Norma.

Michaelsen, L. K., \& Sweet, M. (2008). The essential elements of teambased learning. New directions for teaching and learning, 2008(116), 7-27.
Nicolás, A. S.J., (2010) "Depth, Universality, and Learned Ministry: Challenges to Jesuit Higher Education Today," en Shaping the Future: Networking Jesuit Higher Education for a Globalizing .

Roberson, B., \& Franchini, B. (2014). Effective Task Design for the TBL Classroom. Journal on Excellence in College Teaching, 25. 